\title{
Estrategias didácticas con Scratch para el desarrollo del pensamiento lógico
}

\section{Didactic strategies with Scratch for the development of logical thinking}

Javier José Cevallos Farías. ', Cristopher David Herrera Navas. ${ }^{2}$, Edwin Ludberto Zambrano Vera. ${ }^{3}$ \& Rex Rolando Rumipulla Ramón. ${ }^{4}$

\begin{abstract}
. DOI: https://doi.org/10.33262/concienciadigital.v4i2.1696

Introduction. The development of logical thinking is one of the main skills necessary to develop by students for their insertion into society, since it provides the fundamental tools for conflict resolution, development of critical thinking and analysis of the different casuistry or situations to overcome in different contexts. However, despite its importance, difficulties for its development are still identified. Objective. Strengthen the development of logical thinking through teaching strategies in Scratch in students of the first level of the Software Development career, at the Higher Technological Institute "Los Andes", 2020. Methodology. It has a quantitative approach, a cross-sectional quasi-experimental design and a descriptive scope. The population was considered to be 47 students of the Software Development career of the "Los Andes" Higher Technological Institute. The sample was non-probabilistic for convenience, made up of 18 first-level students. To collect the information, a pretest and posttest were used, which allowed making a comparison between before and after the application of the intervention proposal. Results. The main results showed that students have a low level of development of logical

\footnotetext{
${ }^{1}$ Instituto Tecnológico Superior "Los Andes" y Pontificia Universidad Católica del Ecuador sede Santo Domingo. Santo Domingo, Ecuador. javicevallos@ @istla.edu.ec. Orcid: https://orcid.org/0000-0001-78337461

${ }^{2}$ Pontificia Universidad Católica del Ecuador sede Santo Domingo. Santo Domingo, Ecuador. cdherreran@ @ucesd.edu.ec. Orcid: https://orcid.org/0000-0002-2031-5187

${ }^{3}$ Instituto Tecnológico Superior "Los Andes". Santo Domingo, Ecuador. edwinl.zambrano1 @istla.edu.ec. Orcid: https://orcid.org/0000-0001-5333-1135

${ }^{4}$ Instituto Tecnológico Superior "Los Andes". Santo Domingo, Ecuador. rexr.rumipulla1@istla.edu.ec. Orcid: https://orcid.org/0000-0002-3363-4574.
} 
thinking, however, through the implementation of a didactic strategy in Scratch it was possible to move from a low level to a medium of development of logical thinking, finally by evaluating the impact of the proposal there was a significant improvement in the development of the study variable. Conclution. It can be said that Scratch is a useful tool to strengthen the development of logical thinking, since it contributes to improving verbal reasoning, mathematical reasoning and abstract reasoning.

Keywords: Education, Strategies, Logical Thinking, Reasoning, Software.

\section{Resumen.}

Introducción. El desarrollo del pensamiento lógico es una de las principales competencias necesarias a desarrollar por los estudiantes para su inserción a la sociedad, ya que brinda las herramientas fundamentales para la resolución de conflictos, desarrollo de pensamiento crítico y análisis de las diferentes casuísticas o situaciones a superar en diferentes contextos. Sin embargo, pese a su importancia, aún se identifican dificultades para su desarrollo. Objetivo. Fortalecer el desarrollo del pensamiento lógico mediante estrategias didáctica en Scratch en estudiantes del primer nivel de la carrera Desarrollo de Software, en el Instituto Superior Tecnológico "Los Andes", 2020. Metodología. Tiene un enfoque cuantitativo, diseño cuasiexperimental - transversal y alcance descriptivo. Se consideró como población a 47 estudiantes de la carrera Desarrollo de Software del Instituto Superior Tecnológico "Los Andes". La muestra fue no probabilística por conveniencia conformada por 18 estudiantes del primer nivel. Para el levantamiento de la información se utilizó un pretest y postest, que permitieron hacer una comparación entre el antes y el después de la aplicación de la propuesta de intervención. Resultados. Los principales resultados demostraron que los estudiantes tienen un nivel bajo de desarrollo del pensamiento lógico, sin embargo, a través de la implementación de estrategia didáctica en Scratch se logró transitar de un nivel bajo a un medio de desarrollo de pensamiento lógico, finalmente al evaluar el impacto de la propuesta se registró una mejora significativa en el desarrollo de la variable de estudio. Conclusión. Se puede afirmar que Scratch es una herramienta útil para fortalecer el desarrollo del pensamiento lógico, ya que, contribuye a mejorar el razonamiento verbal, razonamiento matemático y razonamiento abstracto.

Palabras claves: Educación, Estrategias, Pensamiento lógico, Razonamiento, Software.

\section{Introducción.}

En el inicio de la programación una de las tendencias es el uso de herramientas tecnológicas para programar en bloques y con lenguajes visuales, ya que, como menciona Contreras (2018) permiten "iniciar tanto a estudiantes, como a profesores de cualquier nivel educativo en la filosofía o metodología de la programación.” (p.131). Posibilitando, a través de simples procesos, la mejora del pensamiento lógico de los discentes. 
En la formación educativa el pensamiento lógico es de vital importancia, de hecho, Castrillón y Ramírez (2017) manifiestan que: "juega un papel trascendental no solo en el área de matemáticas, sino en todas las demás, pues este provee al estudiante de competencias que le permiten interpretar, deducir, concluir, inferir, razonar, codificar, decodificar, etc" (p.27).

Sin embargo, la Organización de las Naciones Unidas para la Educación Ciencia y Cultura (UNESCO) (2017) muestra que "más de 387 millones de niños en edad escolar que cursan la educación primaria (56\%) y 230 millones de adolescentes que cursan la educación secundaria (61\%) no lograron los niveles mínimos de conocimientos en lógica matemática" (p.2). Los datos reflejan la realidad de la problemática y realza la importancia de trabajar con alternativas didácticas para el desarrollo del pensamiento lógico.

Pero, las falencias del desarrollo de este tipo de pensamiento no solo se pueden analizar desde reportes elaborados por instituciones internacionales, sino que, existe evidencia de su presencia en el continente Latinoamericano, de ello hacen referencia Castrillón y Ramírez (2017) quienes señalan que son preocupantes las deficiencias de pensamiento lógico y estas se dan:

Debido a muchos factores que inciden en el proceso de enseñanza-aprendizaje de los países subdesarrollados, ya que la aplicación de sistemas educativos que no son acorde a la realidad social de los estudiantes, está afectando directamente a la educación en relación. A esto se suma la poca capacitación de los maestros y la asignación insuficiente de recursos económicos destinados a la adquisición de materiales pedagógicos y dispositivos tecnológicos suficientes para lograr aprendizajes significativos. (p. 22)

Estos factores repercuten en la comprensión de problemas planteados por el docente y por ende dificulta el desarrollo de las actividades para resolver los procedimientos adecuadamente por parte de los estudiantes.

Consecutivamente, el problema referente a la dificultad para razonar de forma lógica y abstracta también se puede observar a nivel del Ecuador, según Santana (2016) en el país existe gran cantidad de estudiantes que no han desarrollado su pensamiento lógico, lo que les imposibilita razonar por sí mismos y dificulta el desenvolvimiento en todas las áreas de estudio. Según el autor, esto se debe por la carencia de materiales didácticos para el aprendizaje lógico-matemático, el desinterés de los docentes por aplicar metodologías innovadoras que motiven al estudiante y contenidos abordados que no se relacionan con la realidad. Esto ha creado un perfil del desarrollo del pensamiento lógico deficiente, repercutiendo en las habilidades para la resolución de problemas y en el rendimiento escolar.

Existen diversas investigaciones que destacan la importancia de trabajar el desarrollo del pensamiento lógico a través de las múltiples ventajas que otorgan las TIC. Haciendo referencia a la enseñanza de la programación, se encuentra Ramírez (2019) con el tema; 
"Estrategia didáctica basada en TIC para enseñanza de programación: una alternativa para el desarrollo del pensamiento lógico" (p.1.). Con el objetivo de elaborar una alternativa de estrategia didáctica para la enseñanza de programación mediante recursos multimediales que permitan mejorar el pensamiento lógico, con una metodología anidada, ya que se enmarcó dentro del paradigma cualitativo. En los resultados se obtuvo que, utilizando las TIC, los estudiantes fortalecieron su pensamiento lógico. El estudio concluyó en que el uso de Scratch, en consonancia con las estrategias educativas propuestas, desarrollan el pensamiento lógico. Estos resultados contribuyeron a la formulación de los elementos que componen el pensamiento lógico

Por su parte, otro antecedente a nivel nacional se encuentra elaborado por Cardona y Ramírez (2017) quienes realizaron un estudio denominado: "Desarrollo del pensamiento lógico Matemático apoyado en el uso de blogs en la web 2.0 en los estudiantes de secundaria de la Institución Educativa Real Campestre la Sagrada Familia” (p.1). Con el objetivo de establecer la relación existente entre usar un blog virtual y desarrollar el pensamiento lógico. A partir de una metodología cuasi-experimental, los resultados arrojaron que mejoró el pensamiento lógico cuando mayores eran las estrategias didácticas a partir de los blogs, concluyendo así en que, aplicar las estrategias virtuales y multimedia que ofrecen las TICs contribuye a superar problemas y deficiencias cognitivas de los alumnos. A partir de los postulados planteados por los autores sobre las estrategias didácticas se diseñó la propuesta de intervención de la presente investigación.

Consecutivamente, otro de los estudios a nivel local que tienen en cuenta la importancia del uso de herramientas tecnológicas para el desarrollo del pensamiento es el propuesto por Pérez (2017) con su tema; "uso de Scratch como herramienta para el desarrollo del pensamiento lógico en Programación I” (p.1). A partir del objetivo de identificar el nivel de desarrollo del pensamiento lógico en los estudiantes de Primer Semestre de Filosofía, mediante el recurso Scratch como apoyo didáctico para la mejora profesional, con un diseño investigativo de tipo cuasi-experimental, en los resultados los estudiantes presentaron mejoras en el desarrollo de secuencias y variables, como conclusión el uso de Scratch permitió a los estudiantes fortalecer todos los elementos del pensamiento lógico, aprendiendo así a emplearlo para la solución de conflictos escolares, sociales y familiares. La investigación expuesta contribuyó a la explicación de las posibilidades de Scratch para la programación, los recursos y cada una de sus funcionalidades, esto facilitó la planificación de las clases y la organización de las estrategias.

Con los antecedentes expuestos anteriormente, se evidencia la importancia del desarrollo del pensamiento lógico, los beneficios de usar Scratch e incentivar el mejoramiento de la lógica en los estudiantes. La problemática ha sido abordada por profesionales como docentes en Informática, Ingenieros en Sistemas, con estudios realizados en Europa y América Latina, dichos estudios reflejan que el desarrollo de pensamiento lógico tiene una afectación positiva en el razonamiento y en la construcción del conocimiento. A continuación, se realiza una aproximación onto-epistemológica a los principales conceptos que constituyen la temática abordada. 


\section{Estrategias didácticas.}

Para internalizar la revisión de conceptos referentes a las estrategias didácticas se debe tener en cuenta el criterio de Hernández, Recalde y Luna (2015) quienes afirman que: "tiene que ver con el concepto de aprender a aprender, el aprender de manera autónoma, el aprender de manera significativa, el aprender cooperativamente" (p. 91).

Es decir, se considera a las estrategias didácticas como el elemento fundamental para la preparación previa del proceso de enseñanza-aprendizaje donde el docente establece todos los elementos a utilizar, con el objetivo de propiciar espacios de aprendizaje interactivos, mediante el uso de técnicas pedagógicas, oportunas y eficaces, permitiendo a la sociedad fortalecer el desarrollo de habilidades y destrezas, a partir de su forma de comportarse, actuar y analizar.

Por esta razón, Pita (2019) afirma que para la implementación de estas estrategias es necesario que el docente tenga el conocimiento previo sobre los métodos, recursos y actividades útiles para lograr el aprendizaje óptimo del estudiante, teniendo en cuenta que estas estrategias fortalecen habilidades como: razonar, sintetizar, seleccionar, entre otras, transformándolos en seres capaces de resolver dificultades de la vida cotidiana.

\section{Importancia de las estrategias didácticas.}

La utilización de técnicas y estrategias para el aprendizaje ayudan a generar motivación hacia el estudiante y el uso de nuevas herramientas del docente, es así que para Sotteccani (2018) "la importancia de las estrategias didácticas conlleva a una educación de calidad, ya que ayudan a desarrollar la profesionalidad del docente, y es útil para el aprendizaje del estudiante, quien necesita de una estrategia acorde a sus aptitudes y destrezas" (p. 14). Esto hace referencia que, en el proceso educacional, la utilización de estrategias didácticas forma parte de una visión constructiva de enseñanza para conseguir un aprendizaje eficaz.

Por eso, es importante realizar procesos formativos para el estudiante, mediante herramientas dinámicas e interactivas, las cuales permitan desarrollar el potencial cognitivo, creativo, destrezas, actitudes, capacidades y valores, que ayuden a la profesionalidad del estudiante y además mejoren los procesos de aprendizaje, fortaleciendo el desarrollo integral basado en los principios del constructivismo, fomentando el nivel de desarrollo humano y motivando el interés del estudiante con respecto a una temática (Sotteccani, 2018).

En definitiva, las estrategias didácticas tienen gran importancia en la educación moderna, porque permite desarrollar las habilidades y destrezas del estudiante, mediante la comprensión, reflexión y metacognición en relación a los objetivos planteados.

\section{Aplicación de las estrategias didácticas en los procesos de enseñanza aprendizaje.}

El avance de la ciencia y la tecnología se ha convertido en un pilar principal para la trascendencia social, tanto que, la utilización de estos recursos facilita la interacción entre 
los diversos actores del sistema educativo, fortaleciendo la comunicación y transmisión de contenidos, enfocados a la mejora de la calidad educativa.

De tal manera, que en los últimos años las TIC se han convertido en un eje fundamental en los procesos de enseñanza- aprendizaje, tanto así para el entorno laboral, educativo y personal, no obstante, en la actualidad, cada día se originan innovaciones tecnológicas, por lo que es importante estar a la vanguardia de los diferentes cambios. (Sánchez, García, Steffens y Hernández, 2019, p.8)

En función de lo planteado anteriormente, es importante destacar que en el proceso de enseñanza- aprendizaje las TIC deben ir alineadas con las estrategias didácticas, ya que estas constituyen herramientas pedagógicas que son desarrolladas por el docente, las mismas que se aplican para propiciar entornos de aprendizaje interactivos, fomentando el interés de los estudiantes en la difusión de contenidos, enfocado a nuevas situaciones del entorno.

Del mismo modo, Travieso y Hernández (2017) afirman que la correcta aplicación de estas herramientas didácticas mediados por las TIC, permitirá potenciar el desarrollo completo e integral de los estudiantes, a través de la resolución de problemas, vinculando los conocimientos teóricos con situaciones de la vida práctica, con la finalidad de fortalecer el rendimiento del estudiante de manera efectiva.

\section{Software de programación Scratch.}

El software Scratch es un lenguaje visual y modular que ha sido elaborado por el Grupo Lifelong Kindergarten, de fácil manejo para que los usuarios puedan aprender e interactuar, compartiendo información entre pares, mediante estrategias innovadoras de aprendizaje, para lo cual no es necesario tener conocimientos previos sobre lenguaje de programación.

De esa manera los autores Bolaño y Cuero (2018) definen a esta terminología como un lenguaje de programación visual, dinámico e interactivo que está orientado a promover el aprendizaje de manera fácil a través de herramientas de multimedia e interfaces gráficas.

Por otra parte, el Scratch también motiva al estudiante a pensar, no solo teniendo en cuenta a la lógica, sino también al desarrollo de la creatividad, propiciando así entornos de aprendizaje cooperativo, donde el alumno fomente de forma clara, sencilla e interactiva su capacidad de razonamiento crítico y sistemático.

\section{Características del software de programación Scratch.}

Scratch es un método de aprendizaje que brinda la capacidad de explorar e investigar mediante herramientas de programación visual, apoyado en una interfaz didáctica e interactiva, facilitando la realización y difusión de secuencias animadas con otras personas vía Web. 
Entre las principales características que hacen especial al Scratch podemos mencionar:

- Su uso es fácil y sencillo

- Cuenta con una interfaz interactiva, dinámica y de fácil utilización.

- Tiene una variedad de recursos en el propio programa: objetos, personajes, escenario, sonidos para crear visualizaciones y estilos en sitios Web.

- Facilitan la difusión de proyectos en la Web

- Genera entornos de aprendizaje colaborativos, creando espacios de interacción entre dos o más personas, apoyados en el uso de internet (Vázquez y Ferrer, 2015).

En otras palabras, el uso de esta herramienta permite al estudiante pueda desarrollar programas básicos, donde prevalece el desarrollo de sus habilidades y destrezas, relacionados al contexto laboral y educativo.

\section{Desarrollo del pensamiento lógico.}

Siendo la educación el progreso de un país y la necesidad que tienen los educadores para el desarrollo de la lógica, es de suma importancia aludir a las diferentes dificultades en el aprendizaje de los alumnos, ya que, al utilizar el pensamiento lógico para clasificar, organizar, analizar y concluir la información, es necesario adquirir un razonamiento disciplinado y así alcanzar el pensamiento abstracto, lógico y creativo.

Para Jaramillo y Puga (2016) la educación hoy en día debe atender los diferentes requerimientos de una sociedad, desarrollando así procesos lógicos y abstractos, orientados en la generación de conocimiento para lograr el desempeño de habilidades en un mundo competitivo.

Por su parte, Jaramillo y Puga (2016) expresan que:

El pensamiento lógico es la manera en la cual las personas con especial énfasis, los estudiantes, aprenden a pensar desde edades tempranas o a inicios de la vida escolar, que al ser adecuadamente aplicados desde las aulas permiten llegar a una reflexión significativa. (p.39)

En conclusión, este tipo de pensamiento revela la activación que nace en el cerebro ante la necesidad de razonar de forma lógica, en cuanto a procesos y resultados, a través de la construcción de conocimientos que contribuyan al desarrollo sostenible.

Se ha evidenciado que la temática sobre el desarrollo del pensamiento mediante las TIC ha sido analizada desde diferentes perspectivas, pero no se evidencia una participación activa que involucre a todos los actores del sistema educativo y fortalezca sus competencias y habilidades desde la dimensión didáctica. Por ende, se plantea como objetivo general del presente estudio fortalecer el desarrollo del pensamiento lógico utilizando estrategias didácticas con Scratch en estudiantes del primer nivel de la carrera Desarrollo de Software, en el Instituto Superior Tecnológico "Los Andes”, 2020. 
Además, como procedimientos para la consecución del objetivo general, se plantean los siguientes objetivos específicos:

A. Diagnosticar el nivel de desarrollo del pensamiento lógico a los estudiantes del primer nivel de la carrera Desarrollo de Software;

B. Diseñar una propuesta de intervención basada en estrategias didácticas con Scratch para el desarrollo del pensamiento lógico;

C. Aplicar estrategias didácticas con Scratch para mejorar el desarrollo del pensamiento lógico de los estudiantes del primer nivel de la carrera Desarrollo de Software;

D. Evaluar los resultados del uso de estrategias didácticas con Scratch en el desarrollo del pensamiento lógico de los estudiantes del primer nivel de la carrera Desarrollo de Software.

\section{Metodologia.}

Para el desarrollo de esta investigación se utilizó el enfoque cuantitativo, con base en la consideración de Arenal (2019) quien establece que "basa sus estudios en números estadísticos para dar respuesta a unas causas-efectos concretas” (p. 87). Para analizar y comprobar los resultados obtenidos, se utilizó al diseño cuasi-experimental que modifica la variable independiente para ver su efecto en la dependiente

De igual manera, se aplicó el tipo de investigación de alcance descriptivo, el cual consistió en describir directamente el área objeto de estudio para posteriormente emitir un criterio sobre el comportamiento de los sujetos a investigar. También se utilizó el tipo de investigación aplicada, que según Lozada (2014) "busca la generación de conocimiento con aplicación directa a los problemas de la sociedad (...), ocupándose del proceso de enlace entre la teoría y el producto".

La población considerada para el estudio, está constituida por 47 alumnos de la carrera de Desarrollo de Software. Posteriormente se aplicó el muestreo no probabilístico por conveniencia, seleccionando a 18 alumnos de primer nivel de la carrera de Desarrollo de Software, que cumplían con los siguientes criterios: a) poseer mayores problemas en la aplicación del pensamiento lógico; b) facilidad de acceso para la recolección de la información.

Como técnicas de recolección de datos se utilizó al pretest y postest, teniendo como instrumento al cuestionario propuesto por Padilla (2017) que tenía por objetivo medir el nivel de desarrollo del pensamiento lógico de los estudiantes. En un primer momento (pretest), se diagnosticó el nivel de desarrollo del pensamiento lógico, para a partir de los resultados plantear una propuesta de intervención y aplicarla para después (postest) recoger los resultados obtenidos y evaluar la utilidad de Scratch para el desarrollo del pensamiento lógico. Para efectos del análisis de datos se utilizó la estadística descriptiva e inferencial. 


\section{Resultados y discusión.}

\section{Primer resultado:}

Para la obtención del primer resultado, se optó por aplicar el instrumento elaborado por Padilla (2017) cuya finalidad en el presente estudio fue:

Diagnosticar el nivel de desarrollo del pensamiento lógico a los estudiantes del primer nivel de la carrera Desarrollo de Software.

Para analizar los resultados se va tomar de referencia la siguiente tabla 1:

\begin{tabular}{cc}
\hline ESCALA & VALORACIÓN \\
$\mathbf{1}$ AL $\mathbf{1 0}$ & \\
\hline 1 a 5,9 & Bajo \\
6 a 8,9 & Medio \\
9 a 10 & Alto \\
\hline Tabla 1. Nivel de valoración
\end{tabular}

Fuente: Cevallos, Herrera, Zambrano y Rumipulla (2021)

\section{Análisis de resultados del pretest por indicador}

Se ha tratado de medir fundamentalmente el desarrollo del pensamiento lógico por medio de los indicadores; razonamiento lógico matemático, razonamiento verbal y razonamiento abstracto, obteniéndose los resultados que se aprecian en la tabla 2:

\begin{tabular}{|c|c|c|c|c|c|c|c|c|c|c|c|c|}
\hline \multirow[b]{3}{*}{ 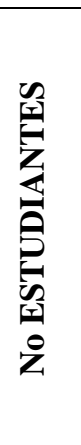 } & \multicolumn{9}{|c|}{ Razonamiento } & \multirow{2}{*}{\multicolumn{3}{|c|}{$\begin{array}{c}\text { Pensamiento Lógico } \\
\text { Resultados }\end{array}$}} \\
\hline & \multicolumn{3}{|c|}{ Matemático } & \multicolumn{3}{|c|}{$\begin{array}{l}\text { Verbal } \\
\stackrel{-}{ }\end{array}$} & \multicolumn{3}{|c|}{$\stackrel{\text { Abstracto }}{\varrho}$} & & & \\
\hline & $\begin{array}{l}6 \\
6 \\
5 \\
5 \\
0 \\
0 \\
0 \\
0 \\
0 \\
0 \\
0\end{array}$ & 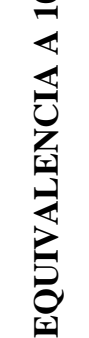 & $\begin{array}{l}z \\
0 \\
0 \\
0 \\
0 \\
0 \\
0 \\
2\end{array}$ & $\begin{array}{l}6 \\
0 \\
5 \\
0 \\
=0 \\
0 \\
0 \\
\vdots \\
0 \\
0 \\
z\end{array}$ & 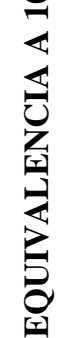 & 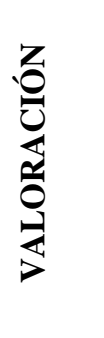 & $\begin{array}{l}6 \\
6 \\
5 \\
0 \\
=0 \\
0 \\
\vdots \\
\vdots \\
0 \\
z\end{array}$ & 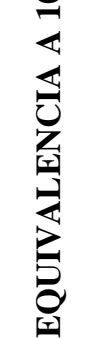 & 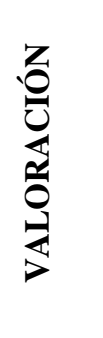 & 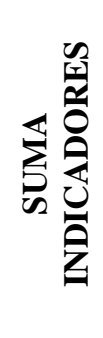 & 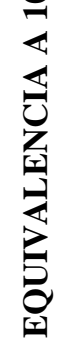 & 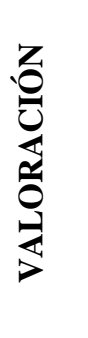 \\
\hline E1 & 2 & 3,33 & Bajo & 0 & 0 & Bajo & 4 & 6,67 & Bajo & 6 & 3,33 & Bajo \\
\hline E2 & 2 & 3,33 & Bajo & 3 & 5 & Bajo & 1 & 1,67 & Bajo & 6 & 3,33 & Bajo \\
\hline E3 & 2 & 3,33 & Bajo & 2 & 3,33 & Bajo & 1 & 1,67 & Bajo & 5 & 2,78 & Bajo \\
\hline E4 & 0 & 0 & Bajo & 0 & 0 & Bajo & 0 & 0 & Bajo & 0 & 0 & Bajo \\
\hline E5 & 2 & 3,33 & Bajo & 2 & 3,33 & Bajo & 1 & 1,67 & Bajo & 5 & 2,78 & Bajo \\
\hline E6 & 3 & 5 & Bajo & 2 & 3,33 & Bajo & 0 & 0 & Bajo & 5 & 2,78 & Bajo \\
\hline E7 & 2 & 3,33 & Bajo & 1 & 1,67 & Bajo & 2 & 3,33 & Bajo & 5 & 2,78 & Bajo \\
\hline E8 & 3 & 5 & Bajo & 2 & 3,33 & Bajo & 1 & 1,67 & Bajo & 6 & 3,33 & Bajo \\
\hline E9 & 2 & 3,33 & Bajo & 1 & 1,67 & Bajo & 0 & 0 & Bajo & 3 & 1,67 & Bajo \\
\hline E10 & 0 & 0 & Bajo & 1 & 1,67 & Bajo & 3 & 5 & Bajo & 4 & 2,22 & Bajo \\
\hline E11 & 1 & 1,67 & Bajo & 2 & 3,33 & Bajo & 0 & 0 & Bajo & 3 & 1,67 & Bajo \\
\hline E12 & 2 & 3,33 & Bajo & 2 & 3,33 & Bajo & 0 & 0 & Bajo & 4 & 2,22 & Bajo \\
\hline E13 & 1 & 1,67 & Bajo & 2 & 3,33 & Bajo & 0 & 0 & Bajo & 3 & 1,67 & Bajo \\
\hline E14 & 0 & 0 & Bajo & 2 & 3,33 & Bajo & 1 & 1,67 & Bajo & 3 & 1,67 & Bajo \\
\hline
\end{tabular}




\begin{tabular}{lcccccccccccc}
\hline E15 & 2 & 3,33 & Bajo & 2 & 3,33 & Bajo & 0 & 0 & Bajo & 4 & 2,22 & Bajo \\
E16 & 1 & 1,67 & Bajo & 2 & 3,33 & Bajo & 1 & 1,67 & Bajo & 4 & 2,22 & Bajo \\
E17 & 2 & 3,33 & Bajo & 2 & 3,33 & Bajo & 2 & 3,33 & Bajo & 6 & 3,33 & Bajo \\
E18 & 2 & 3,33 & Bajo & 1 & 1,67 & Bajo & 0 & 0 & Bajo & 3 & 1,67 & Bajo \\
MEDIA & $\mathbf{1 , 6}$ & $\mathbf{2 , 6 8}$ & Bajo & $\mathbf{1 , 6 1}$ & $\mathbf{2 , 6 8}$ & Bajo & $\mathbf{0 , 9 4}$ & $\mathbf{1 , 5 7}$ & Bajo & $\mathbf{4 , 1 7}$ & $\mathbf{2 , 3 2}$ & Bajo \\
\hline
\end{tabular}

Fuente: Cevallos, Herrera, Zambrano y Rumipulla (2021)

El indicador razonamiento matemático obtuvo una media de 2,68/10, mientras el indicador de razonamiento verbal obtuvo una media 2,68/10, finalmente el indicador razonamiento abstracto obtuvo una media de 1,57/10. Al hacer un análisis global comparando las medias de cada indicador se puede observar que existe un bajo nivel en el desarrollo de las habilidades del pensamiento lógico, esto significa que los estudiantes presentan problemas para resolver problemas que requieren de razonamiento numérico (razonamiento matemático), conflictos que precisan análisis e interpretación (razonamiento abstracto) y tienen dificultad para razonar, deducir, codificar y decodificar información de todas las áreas del saber (razonamiento verbal).

\section{Segundo resultado:}

Diseñar una propuesta de intervención basada en estrategias didácticas con Scratch para el desarrollo del pensamiento lógico.

\section{Diseño de la propuesta}

La integración de recursos, las herramientas para aplicar y las orientaciones para el uso de estrategias didácticas con Scratch, permiten concretar un escenario de aprendizaje para el desarrollo del pensamiento lógico. La propuesta está basada en las Tecnólogas de la Información y de la Comunicación (TICs), a través de 10 clases planificadas y distribuidas en cinco unidades, que son prácticas de dificultad incremental, siendo el estudiante el actor principal. Cada clase proyecta a desarrollar los indicadores en estudio planteados y responde a una serie de logros esperados definidos.

La propuesta se realizó a través de un conjunto de estrategias y actividades para mejorar el nivel del pensamiento lógico utilizando la aplicación SCRATCH. Las actividades se han diseñado para permitir paulatinamente familiarizarse con el software e ir adquiriendo las habilidades del pensamiento lógico. En concreto, las actividades animan a la exploración de razonamiento matemático, verbal y abstracto que son elementos fundamentales para desarrollar este tipo de pensamiento. A continuación, se describirá cada uno de las unidades, su composición y logros esperados:

Primera unidad - IDE Scratch 4.0, explorando: esta unidad está conformada por tres clases, que parte desde la instalación del IDE Scratch, la socialización con su entorno, los primeros pasos y el reconocimiento de cada una de sus áreas. También se trabajarán los primeros bloques del software, que son: de movimiento, entre posiciones, distanciamiento, direccionamiento y de control. Los logros esperados se centran en 
identificar correctamente las herramientas del programa, aprender a navegar por el mismo y posicionar mediante los bloques objetos en coordenadas específicas logrando controlar los movimientos.

Segunda unidad - Animaciones: esta unidad posee tres clases que tienen como finalidad el trabajo con bucles, bloques de apariencia, sensores y de control. Así también se abordará la creación de clones y de objetos. Los logros esperados que se establecen para esta unidad se centrar en la generación de objetos autónomos que respondan a los principios de la inteligencia artificial mediante bucles y que posean funcionalidad en diferentes aplicaciones. Del mismo modo, se pretende que los estudiantes mejoren apariencias de escenarios, creen diálogos de bienvenida para interactuar con el usuario, manejen objetos repetitivos con clones y secuencias e implementen funciones y métodos que mejoren la estructura de una aplicación.

Tercera unidad - Historias: esta unidad se conforma de una clase centrada en la utilización del bloque de apariencia, donde se enseña a implementación de diálogos, cambiar fondos, esconder y mostrar objetos y a usar el sub-bloque de pensar y decir. De igual manera, a que diseñen e implementen pantallas de inicio y finalización para los juegos. Los logros esperados son que los estudiantes recreen historias que puedan dar pauta a crear un juego o aplicaciones, de igual manera, la capacidad de editar y diseñar personales propios con sus escenarios.

Cuarta unidad - Juegos: esta unidad consta de dos clases, donde se aborda la creación de scrollers, que consiste en la fijación de objetos. De igual manera, a manejar gravedad con objetos, teniendo en cuenta: operadores relacionales, aritméticos, creación y edición de escenarios y la creación de juegos con scroll horizontal. De igual manera, se trabaja con scroll en pantalla vertical y la implementación de clones y ciclos repetitivos mediante recursos multimedia.

Quinta unidad - Proyecto final: esta unidad consta de una clase donde se realizará un juego completo implementando todos los temas abordados en las unidades anteriores. Se tiene que tener en cuenta los siguientes elementos: pantalla de inicio, pantalla de ejecución, sonidos de objetos, sumatoria de puntos, cambios de disfraces, cambio de escenarios, llamado de funciones. Como logros, se esperaba que los estudiantes creen juegos propios con niveles de dificultad, puntajes, vidas, funciones, diálogos y pantallas interactivas que combine los recursos audiovisuales.

\section{Tercer resultado:}

* Aplicar estrategias didácticas con Scratch para mejorar el desarrollo del pensamiento lógico de los estudiantes del primer nivel de la carrera Desarrollo de Software.

A continuación, se evidencia las ponderaciones y calificaciones de las diferentes actividades que se evaluaron a lo largo de las clases programadas: 
ISSN: 2600-5859

\begin{tabular}{cclc}
\hline UNIDAD & CLASES & INDICADOR & EVALUACIÓN \\
\hline \multirow{2}{*}{$\mathbf{1}$} & 1 & Razonamiento Matemático & 4,60 \\
& 2 & Razonamiento Verbal & 3,60 \\
& 3 & Razonamiento Abstracto & 3,30 \\
$\mathbf{2}$ & 4 & Razonamiento Matemático & 4,50 \\
& 5 & Razonamiento Verbal & 3,70 \\
$\mathbf{3}$ & 6 & Razonamiento Abstracto & 4,70 \\
& 7 & Razonamiento Matemático & 6,20 \\
$\mathbf{4}$ & 8 & Razonamiento Verbal & 5,70 \\
& 9 & Razonamiento Abstracto & 5,20 \\
& & MEDIA & $\mathbf{4 , 6 2}$ \\
\hline
\end{tabular}

Tabla 3. Resultado general de actividades

Fuente: Cevallos, Herrera, Zambrano y Rumipulla (2021)

En las evaluaciones de actividades por clase y cada práctica que se realizó se evidencia que los estudiantes van incrementando paulatinamente el razonamiento lógico, razonamiento verbal, razonamiento abstracto, y con motivación alcanzando la destreza de pensamiento lógico y los logros esperados planteados.

\begin{tabular}{lcc}
\hline INDICADOR & MEDIA & VALORACIÓN \\
\hline Razonamiento Matemático & 5,10 & Medio \\
Razonamiento Verbal & 4,34 & Medio \\
Razonamiento Abstracto & 4,40 & Medio \\
PROMEDIO GENERAL & 4,62 & Medio \\
\hline
\end{tabular}

Tabla 4. Resultados de actividades por indicador.

Fuente: Cevallos, Herrera, Zambrano y Rumipulla (2021)

En el proceso de la intervención los estudiantes demostraron interés en cada clase, actividad y problema propuesto, como se evidencia en la tabla 4 que el razonamiento matemático con una media de 5,10/10, razonamiento verbal con una media de 4,34/10, razonamiento abstracto con una media de 4,62/10. De acuerdo a los resultados obtenidos se evidencia una mejora significativa en el desarrollo del pensamiento lógico.

\section{Cuarto resultado:}

Evaluar los resultados del uso de estrategias didácticas con Scratch en el desarrollo del pensamiento lógico de los estudiantes.

\section{Análisis de resultados del postest por indicador}

Después de la aplicación de la propuesta para la mejora del desarrollo del pensamiento lógico teniendo en cuenta los siguientes indicadores; razonamiento lógico matemático, razonamiento verbal y razonamiento abstracto, se obtuvieron los siguientes resultados: 
Resultados

\begin{tabular}{lcccccccccccc}
\hline E1 & 4 & 6,67 & Medio & 5 & 8,33 & Medio & 5 & 8,33 & Medio & 14 & 7,78 & Medio \\
E2 & 5 & 8,33 & Medio & 5 & 8,33 & Medio & 4 & 6,67 & Medio & 14 & 7,78 & Medio \\
E3 & 5 & 8,33 & Medio & 5 & 8,33 & Medio & 3 & 5,00 & Bajo & 13 & 7,22 & Medio \\
E4 & 4 & 6,67 & Medio & 3 & 5 & Bajo & 2 & 3,33 & Medio & 9 & 5 & Bajo \\
E5 & 5 & 8,33 & Medio & 4 & 6,67 & Medio & 4 & 6,67 & Medio & 13 & 7,22 & Medio \\
E6 & 5 & 8,33 & Medio & 4 & 6,67 & Medio & 3 & 5,00 & Bajo & 12 & 6,67 & Medio \\
E7 & 4 & 6,67 & Medio & 3 & 5 & Bajo & 4 & 6,67 & Medio & 11 & 6,11 & Medio \\
E8 & 6 & 10 & Alto & 5 & 8,33 & Medio & 4 & 6,67 & Medio & 15 & 8,33 & Medio \\
E9 & 4 & 6,67 & Medio & 3 & 5 & Bajo & 3 & 5,00 & Bajo & 10 & 5,56 & Bajo \\
E10 & 5 & 8,33 & Medio & 3 & 5 & Bajo & 4 & 6,67 & Medio & 12 & 6,67 & Medio \\
E11 & 5 & 8,33 & Medio & 4 & 6,67 & Medio & 4 & 6,67 & Medio & 13 & 7,22 & Medio \\
E12 & 5 & 8,33 & Medio & 5 & 8,33 & Medio & 5 & 8,33 & Medio & 15 & 8,33 & Medio \\
E13 & 4 & 6,67 & Medio & 3 & 5 & Bajo & 3 & 5,00 & Bajo & 10 & 5,56 & Bajo \\
E14 & 6 & 10 & Medio & 5 & 8,33 & Medio & 4 & 6,67 & Medio & 15 & 8,33 & Medio \\
E15 & 5 & 8,33 & Medio & 5 & 8,33 & Medio & 5 & 8,33 & Medio & 15 & 8,33 & Medio \\
E16 & 5 & 8,33 & Medio & 3 & 5 & Medio & 4 & 6,67 & Medio & 12 & 6,67 & Medio \\
E17 & 5 & 8,33 & Medio & 4 & 6,67 & Medio & 4 & 6,67 & Medio & 13 & 7,22 & Medio \\
E18 & 3 & 5 & Bajo & 3 & 5 & Bajo & 4 & 6,67 & Medio & 10 & 5,56 & Bajo \\
MEDIA & $\mathbf{4 , 7 3}$ & $\mathbf{7 , 8 7}$ & Medio & $\mathbf{4}$ & $\mathbf{6 , 6 7}$ & Medio & $\mathbf{3 , 8 4}$ & $\mathbf{6 , 3 9}$ & Medio & $\mathbf{1 2 , 5 6}$ & $\mathbf{6 , 9 8}$ & Medio \\
\hline
\end{tabular}

Tabla 5. Resultados del postest por indicador

Fuente: Cevallos, Herrera, Zambrano y Rumipulla (2021)

Se evidencia 4 estudiantes que no superan la ponderación baja, pero que en comparación al pretest existe un incremento, por ende, se desarrolló el pensamiento lógico.

\section{Resultado general por indicador del postest}

\begin{tabular}{lcc}
\hline INDICADOR & MEDIA & VALORACIÓN \\
\hline Razonamiento Matemático & 7,87 & Medio \\
Razonamiento Verbal & 6,67 & Medio \\
Razonamiento Abstracto & 6,39 & Medio \\
MEDIA GENERAL & $\mathbf{6 , 9 8}$ & Medio \\
\hline
\end{tabular}

Tabla 6. Resultado general por indicador del postest

Fuente: Cevallos, Herrera, Zambrano y Rumipulla (2021)

El indicador razonamiento matemático obtuvo una media de 7,87/10, mientras el indicador de razonamiento verbal obtuvo una media 6,67/10, finalmente el indicador razonamiento abstracto obtuvo una media de 6,39/10. Al hacer un análisis global 
comparando las medias de cada indicador se puede observar que a raíz de la intervención el nivel de desarrollo de pensamiento lógico aumentó de manera significativa.

\section{Balance general de resultados}

\begin{tabular}{lccc}
\hline INDICADORES & PRETEST & INTERVENCIÓN & POSTEST \\
\hline Razonamiento Matemático & 2,68 & 5,10 & 7,87 \\
Razonamiento Verbal & 2,68 & 4,34 & 6,67 \\
Razonamiento Abstracto & 1,58 & 4,40 & 6,39 \\
MEDIAS FINALES & $\mathbf{2 , 3 2}$ & $\mathbf{4 , 6 2}$ & $\mathbf{6 , 9 8}$ \\
\hline
\end{tabular}

Tabla 7. Resultado general en contraste con los tres resultados de la investigación Fuente: Cevallos, Herrera, Zambrano y Rumipulla (2021)

En contraste de los resultados obtenidos se puede establecer que el nivel de avance en el desarrollo del pensamiento lógico ha mejorado, en un inicio la media del pretest fue de 2,32/10 (nivel bajo); ya en la intervención se observó un incremento con una media de 4,62/10 (nivel bajo), finalmente en el postest se evidenció una media de 6,98/10 (nivel medio), registrándose un incremento significativo de 4,66 puntos entre el pretest y el postest, estos resultados demuestran que la aplicación de estrategias didácticas con Scratch mejora el desarrollo de las habilidades del pensamiento lógico.

\section{Comprobación de la hipótesis:}

Luego de analizar los resultados obtenidos, se comprueba la hipótesis de investigación planteada mediante la medida no paramétrica de chi2, obteniendo los siguientes resultados:

\begin{tabular}{llcccccccc}
\hline & \multicolumn{9}{c}{ Resultados_POSTEST } \\
& & 5,00 & 5,56 & 6,12 & 6,67 & 7,23 & 7,78 & 8,34 & Total \\
\hline Resultados_PRETEST &, 00 & 1 & 0 & 0 & 0 & 0 & 0 & 0 & 1 \\
& 1,67 & 0 & 3 & 0 & 0 & 1 & 0 & 1 & 5 \\
& 2,23 & 0 & 0 & 0 & 2 & 0 & 0 & 2 & 4 \\
& 2,78 & 0 & 0 & 1 & 1 & 2 & 0 & 0 & 4 \\
Total & 3,34 & 0 & 0 & 0 & 0 & 1 & 2 & 1 & 4 \\
& & 1 & 3 & 1 & 3 & 4 & 2 & 4 & 18
\end{tabular}

Prueba de $\mathrm{Chi}^{2}$

Nivel de significancia asintótica bilateral: 0,006

Tabla 8. Tabla cruzada y prueba de chi2 de los resultados entre el Pretest y Postest

Fuente: Cevallos, Herrera, Zambrano y Rumipulla (2021)

Los datos presentados en la tabla 8, permiten divisar que los resultados del pretest se relacionan con los del postest, identificando que, los estudiantes que obtuvieron las notas más altas en el pretest (rango de 2,78 - 3,34) obtienen las más altas en el postest (rango de desde 6 a 8,35). Esto permite inferir en que, a mayor uso de estrategias didácticas con Scratch, mayor será el desarrollo del pensamiento lógico.

Finalmente, a partir del estadístico de chi2 se obtiene el siguiente nivel de significancia asintótica bilateral (NSAB): 0,006. Conforme a lo que establece Monterrey y GómezRestrepo (2007) en este estadístico cuando el NSBA es > 0,05 se rechaza la hipótesis de investigación y se acepta la nula, mientras que, cuando es $<0,05$ se acepta la de investigación, que es el caso del presente estudio, por lo tanto, se comprueba que: 
- Hi: Las estrategias didácticas en Scratch fortalecen el pensamiento lógico en los estudiantes.

También, de forma descriptiva, los resultados comparativos entre los promedios del pretest y postest, luego de aplicar estrategias didácticas con Scratch, presentan una mejora $46,6 \%$, siendo significativa y respaldando a la prueba inferencial realizada anteriormente.

A continuación, se realizará una discusión entre los resultados obtenidos en la presente investigación con los hallazgos de autores que trabajaron bajo la misma línea de estudio. Se trata de buscar concordancias, contraposiciones, similitudes, limitaciones e implicaciones teóricas y prácticas.

Como primer resultado se diagnosticó que los estudiantes tienen un nivel bajo de desarrollo del pensamiento lógico, siendo el indicador razonamiento abstracto en el que mayores problemas presentan los estudiantes. Esto se relaciona con lo enunciado por la UNESCO (2017) quien manifiesta que "más de 387 millones de niños en edad escolar que cursan la educación primaria (56\%) y 230 millones de adolescentes que cursan la educación secundaria (61\%) no lograron los niveles mínimos de conocimientos en lógica matemática" (p.2). Pese a que, Cardona y Ramírez (2017) manifiesta que el pensamiento lógico juega un papel trascendental no sólo enfrascado en el área de las matemáticas, sino en todas las demás, ya que apoya a interpretar, razonar, deducir, codificar y decodificar cualquier tipo de información en todas las áreas. De igual manera lo plantea Azoulay (2020) quien resalta que, el pensamiento lógico es un ámbito con mayor deficiencia, pero vital para la sociedad contemporánea para el desarrollo económico y social del mundo actual.

El segundo resultado permitió el diseño de una propuesta de intervención basada en estrategias didácticas con Scratch, a partir de las tres dimensiones que conforman el pensamiento lógico, es decir: el razonamiento verbal, matemático y abstracto. Para ello se diseñó una propuesta de diez clases que abordan cinco unidades divididas en diez temas. Asimismo, se plateó las destrezas a alcanzar, los recursos a utilizar, las actividades a realizar, los logros esperados e indicadores alcanzados por cada área del pensamiento lógico. Esto se relaciona con lo planteado por Sánchez, García, Steffens y Hernández (2019) quienes aluden al hecho de que una estrategia didáctica debe poseer una planificación de trasfondo que declare los elementos rectores y orientadores de las actividades, tales como: destrezas e indicadores de logro.

De igual manera, la importancia del uso de herramientas interactivas como SCRATCH para el planteamiento de estrategias didácticas, también la argumenta Sotteccani (2018) mencionado que, este tipo de herramientas dinámicas e interactivas permiten desarrollar de forma integral al estudiante a partir de los principios del constructivismo. Del mismo modo concuerda Penalva (2018) mencionando que el diseño de estrategias didácticas no se puede trabajar sin tener en cuenta la sistematicidad de los elementos.

En relación al tercer resultado, se aplicó estrategias didácticas con Scratch para mejorar el desarrollo del pensamiento lógico, obteniendo así que los estudiantes se enmarcan en 
un nivel medio de desarrollo, con un promedio general de 4,62, presentando aún problemas con el razonamiento abstracto, sin embargo, se evidenció una mejora significativa a partir de la aplicación de la propuesta. Estos resultados concuerdan con los obtenidos por Pérez (2017) quien concluye en que la herramienta Scratch fortalece el pensamiento lógico de los estudiantes y facilita la resolución de problemas en todos los entornos. De manera similar, Bolaño y Cuero (2018) enuncian que Scratch está orientado a promover el aprendizaje de mantera fácil a través de herramientas multimedia e interfaces gráficas. Esta afirmación se ratifica con lo plateado por Vázquez y Ferrer (2015) señalando que el software en cuestión es de fácil uso, con interfaz interactiva y tiene una gran gama de recursos, tales como: objetos, personales, escenarios y efectos multimedia, además de permitir espacios de interacción entre dos o más personas a la vez.

Finalmente, se evaluó los resultados del uso de estrategias didácticas con Scratch para el desarrollo de pensamiento lógico, obteniendo resultados significativos, ya que luego de la propuesta de intervención el postest arrojó un incremento en el promedio general que lo enmarca en un nivel de desarrollo medio, equivalente a 6,98, que en relación al pretest alcanzó una media de 2,32, en definitiva se estableció un porcentaje de mejora de 46,6\%, lo cual respalda las estrategias planteadas como útiles para el desarrollo del pensamiento lógico. Esto concuerda con los resultados obtenidos por Ramírez (2019) quien menciona que Scratch contribuye al desarrollo del pensamiento lógico de forma significativa, al igual que Cardona y Ramírez (2017) que mencionan que las estrategias desarrolladas en este tipo de software contribuyen a superar las deficiencias cognitivas en alumnos.

\section{Conclusiones.}

- Se diagnosticó que el nivel de desarrollo del pensamiento lógico de los estudiantes es bajo, presentando mayores dificultades en el área del razonamiento abstracto, esto refleja que presentan inconvenientes en la resolución de problemas de tipo lógico, la reflexión crítica ante diversas situaciones, la autonomía para el aprendizaje, la creatividad e innovación y la toma de decisiones acertadas.

- Se diseñó una propuesta de intervención basada en estrategias didácticas con Scratch para el desarrollo del pensamiento lógico, la cual permitió percibir que, para el planteamiento sistemático de actividades es necesario primero realizar un reconocimiento de las capacidades de los estudiantes y su punto de partida, de esta forma, se puede establecer un diagnóstico que permita plantear de forma asertiva los mecanismos de control y orientación del proceso de enseñanza-aprendizaje, tales como: objetivos o destrezas a desarrollar y logros e indicadores a alcanzar por cada área que se trabaje.

- Mediante la aplicación estrategias didácticas con Scratch para mejorar el desarrollo del pensamiento lógico, se logra resultados significativos en la estimulación del razonamiento verbal, razonamiento matemático, presentando algunos problemas persistentes con el pensamiento abstracto. A partir de estos resultados, se puede inferir que se requiere un tiempo mayor de aplicación para aumentar la significatividad de los resultados, asimismo, es necesario enfatizar en 
el trabajo para mejorar el desarrollo del razonamiento abstracto, ya que presenta mayores dificultades para los estudiantes.

- Se puede establecer que el uso de estrategias didácticas con Scratch permite un desarrollo del pensamiento lógico significativo, ya que, permitió transitar de un nivel bajo a un nivel medio de desarrollo del pensamiento lógico matemático con un porcentaje de mejora del 46,6\%. Esto refleja que las estrategias didácticas planteadas mediante la herramienta en cuestión sirven para generar motivación e implicación en el aprendizaje, por ende, se considera prioritario, en la realidad actual, utilizar los medios tecnológicos para la potenciación de los procesos educativos y así cambiar el paradigma educativo utilizado en la realidad actual, donde los docentes son protagonistas y los estudiantes meros receptores.

\section{Referencias bibliográficas.}

Arenal, L. (2019). Tratamiento y Análisis de la Información de Mercados. UF1781. La Rioja: Tutor Formación.

Bolaño, M., y Cuero, E. (2018). Uso deI software educativo Scratch como herramienta para el desarrollo deI pensamiento variaciona I. Española: Editorial Académica Española. Recuperado: https://bit.ly/2ZekHNH

Castrillón, C. y Ramirez, P. (2017). Desarrollo del pensamiento Lógico Matemático apoyado en el uso de blogs en la web 2.0 en los estudiantes de secundaria de la Institución Educativa Real Campestre La Sagrada Familia sede principal del Municipio de Fresno-Tolima 2013-2014. Obtenido de https://bit.ly/3gJsU6K

Contreras, E. (2018). Software para iniciar tanto a estudiantes, profesores de cualquier nivel educativo en la filosofía o metodología de la programación. Recuperado de: https://bit.ly/2S7afHE

García, G. (2017). Proyecto pedagógico y aprendizaje cooperativo: Scratch y nutrición. Recuperado de: https://upcommons.upc.edu/handle/2117/107697

Hernández, I., Recalde, J., y Luna, J. (2015). Estrategia Didáctica: una competencia docente en la formación para el mundo laboral. Revista Latinoamericana de Estudios Educativos, 11(2), 73-94. Recuperado de: https://bit.ly/2QAzWzU

Hernández, R., Fernández, C., y Baptista, L. (2014). Metodología de la Investigación (Punta Santa Fe C.P. 01376 Sexta Edición ed.). México: McGRAW-HILL / INTERAMERICANA.

Jaramillo, L., y Puga, L. (2016). El pensamiento lógico-abstracto como sustento para potenciar los procesos cognitivos en la educación. Revista Científica de America Latina, 21(5), 31-55. Recuperado de: https://bit.ly/3eCj9nY 
Monterrey, P. y Gómez, R. (2007). Aplicación de las pruebas de hipótesis en la investigación en salud: ¿estamos en lo correcto? Universidad Medica, 48(3), 193 206. Recuperado de: https://www.redalyc.org/pdf/2310/231018668002.pdf

Padilla, I. (2017). Instructivo Prueba de Aptitud Académica. Recuperado de: https://bit.ly/2SasADF

Pérez, N. (2017). Uso de SCRATCH como herramienta para el desarrollo del pensamiento computacional en Programación I de la carrera de Informática de la Universidad Central del Ecuador. Recuperado de: https://bit.ly/3vpaEUg

Pita, L. (2019). Estrategias didácticas en el desarrollo de habilidades investigativas. (L. y. Universidad de Guayaquil. Facultad de Filosofía, Ed.). Recuperado de: http://repositorio.ug.edu.ec/handle/redug/40563

Ramírez, W. (2019). Estrategia didáctica basada en TIC para enseñanza de Programación: Recuperado de https://repositorio.uptc.edu.co/bitstream/001/2946/1/TGT_1548.pdf

Ramírez, Y. (2019). Estrategia didáctica basada en TIC para enseñanza de programación: una alternativa para el desarrollo del pensamiento lógico. Recuperado de https://repositorio.uptc.edu.co/bitstream/001/2946/1/TGT_1548.pdf

Sánchez, M., García, J., Steffens, E., y Hernández, H. (2019). Estrategias Pedagógicas en Procesos de Enseñanza y Aprendizaje en la Educación Superior incluyendo Tecnologías de la Información y las Comunicaciones. Información tecnológica, 30(3), 1-15. Recuperado de: https://bit.ly/2QA4m5i

Santana, C. (2016). Estrategias didácticas y el desarrollo del pensamiento lógico matemático de los niños y las niñas de quinto año del paralelo " $B$ " de la unidad educativa Cristóbal Colón de la parroquia Atahualpa del cantón Ambato, provincia de Tungurahua. Tesis de grado: Universidad Técnica de Ambato.

Sotteccani, C. (2018). Influencia de las estrategias didactivas en el aprendizaje signiticativo del estudiante. Recuperado de https://bit.ly/3tW2GkY

Travieso, D. y Hernández, A. (enero de 2017). El desarrollo del pensamiento lógico a través del proceso enseñanza-aprendizaje. Cubana de Educación Superior, 36(1). Recuperado de: https://bit.ly/3aKpZqj

UNESCO. (2017). More Than One-Half of Children and Adolescents Are Not Learning Worldwide. Recuperado de https://bit.ly/3aHd8Fi 
Vázquez, E. y Ferrer, D. (2015). La creación de videojuegos con Scratch en Educación Secundaria. Communication papers: media literacy and gender studies, 4(6), 6373. 
PARA CITAR EL ARTÍCULO INDEXADO.

Cevallos Farías, J. J., Herrera Navas, C. D., Zambrano Vera, E. L., \& Rumipulla Ramón, R. R. (2021). Estrategias didácticas con Scratch para el desarrollo del pensamiento lógico . ConcienciaDigital, 4(2), 306-325. https://doi.org/10.33262/concienciadigital.v4i2.1696

\section{Liencia}

El artículo que se publica es de exclusiva responsabilidad de los autores y no necesariamente reflejan el pensamiento de la Revista Conciencia Digital.

El artículo queda en propiedad de la revista y, por tanto, su publicación parcial y/o total en otro medio tiene que ser autorizado por el director de la Revista Conciencia Digital.

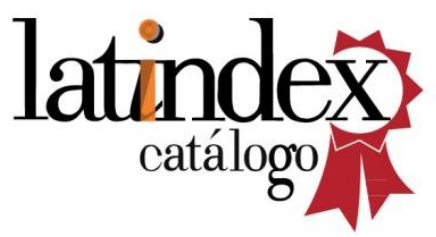

